\title{
Long QT Interval in Turner Syndrome - A High Prevalence of LQTS Gene Mutations
}

\author{
Christian Trolle', Kristian H. Mortensen ${ }^{1,2}$, Lisbeth N. Pedersen ${ }^{3}$, Agnethe Berglund ${ }^{1}$, Henrik K. Jensen ${ }^{4}$, \\ Niels H. Andersen ${ }^{4}$, Claus H. Gravholt ${ }^{1,3 *}$
}

1 Department of Endocrinology and Internal Medicine and Medical Research Laboratories, Aarhus University Hospital, Aarhus, Denmark, 2 Department of Radiology, Cambridge University Hospitals, Cambridge, United Kingdom, 3 Department of Molecular Medicine, Aarhus University Hospital, Aarhus, Denmark, 4 Department of Cardiology, Aarhus University Hospital, Aarhus, Denmark

\begin{abstract}
Objectives: QT-interval prolongation of unknown aetiology is common in Turner syndrome. This study set out to explore the presence of known long QT mutations in Turner syndrome and to examine the corrected QT-interval (QTc) over time and relate the findings to the Turner syndrome phenotype.

Methods: Adult women with Turner syndrome $(n=88)$ were examined thrice and 68 age-matched healthy controls were examined once. QTc was measured by one blinded reader (intra-reader variability: $0.7 \%$ ), and adjusted for influence of heart rate by Bazett's (bQTc) and Hodges's formula (hQTc). The prevalence of mutations in genes related to Long QT syndrome was determined in women with Turner syndrome and a QTc $>432.0$ milliseconds (ms). Echocardiographic assessment of aortic valve morphology, 24-hour blood pressures and blood samples were done.

Results: The mean hQTc in women with Turner syndrome $(414.0 \pm 25.5 \mathrm{~ms})$ compared to controls (390.4 $\pm 17.8 \mathrm{~ms})$ was prolonged $(p<0.001)$ and did not change over time $(416.9 \pm 22.6$ vs. $415.6 \pm 25.5 \mathrm{~ms} ; \mathrm{p}=0.4) .45, X$ karyotype was associated with increased hQTc prolongation compared to other Turner syndrome karyotypes (418.2 \pm 24.8 vs. $407.6 \pm 25.5 \mathrm{~ms}$; $\mathrm{p}=0.055)$. In women with Turner syndrome and a bQTc $>432 \mathrm{~ms}, 7$ had mutations in major Long QT syndrome genes (SCN5A and $\mathrm{KCNH} 2$ ) and one in a minor Long QT syndrome gene (KCNE2).
\end{abstract}

Conclusion: There is a high prevalence of mutations in the major LQTS genes in women with TS and prolonged QTc. It remains to be settled, whether these findings are related to the unexplained excess mortality in Turner women.

Clinical Trial Registration: NCT00624949. https://register.clinicaltrials.gov/prs/app/action/SelectProtocol/sid/S0001FLI/ selectaction/View/ts/3/uid/U000099E.

Citation: Trolle C, Mortensen KH, Pedersen LN, Berglund A, Jensen HK, et al. (2013) Long QT Interval in Turner Syndrome - A High Prevalence of LQTS Gene Mutations. PLoS ONE 8(7): e69614. doi:10.1371/journal.pone.0069614

Editor: Katriina Aalto-Setala, University of Tampere, Finland

Received February 8, 2013; Accepted June 7, 2013; Published July 25, 2013

Copyright: (c) 2013 Trolle et al. This is an open-access article distributed under the terms of the Creative Commons Attribution License, which permits unrestricted use, distribution, and reproduction in any medium, provided the original author and source are credited.

Funding: Funding from the Danish Ministry for Science and Technology and Innovation, The Danish Heart Foundation, Novo Nordisk Foundation, Aase og Ejnar Danielsen Foundation, Korning Foundation, Hede Nielsens Foundation, Eva og Henry Frænkels Minde Foundation and Snedkermester Sophus Jacobsen og hustru Astrid Jacobsens Foundation. The funders had no role in study design, data collection and analysis, decision to publish, or preparation of the manuscript.

Competing Interests: The authors have declared that no competing interests exist.

*E-mail: ch.gravholt@dadlnet.dk

\section{Introduction}

The corrected QT interval (QTc-interval) is prolonged in 33\% of children and $20 \%$ of adults with Turner syndrome (TS) [1-3]. A prolonged QTc-interval is associated with increased risk of sudden death in the general population [4]. Long QT syndrome (LQTS) in general is characterized by prolongation of the QT-interval in association with the risk of Torsade de Point. LQTS comprises an acquired [5] as well as a congenital form [6]. However, in TS the mechanism behind the QTc prolongation is unknown. Women with TS characteristically display growth retardation with reduced final height and hypogonadism with infertility requiring hormone replacement therapy (HRT) [7]. Increased morbidity and mortality are present due to congenital heart disease, ischemic heart disease, hypertension, and diabetes [8-10]. A relative sinus tachycardia is a life-long phenomenon [1,2], where conduction of the electrical impulse through the atria and atrioventricular node is accelerated [2], and the risk of atrial tachycardia may be increased [11] whereas bradycardia is rare [12]. Whether a QTcinterval prolongation contributes to the increased excess mortality in women with TS remains to be solved [9].

We therefore set out to examine the QTc-interval in women with TS assessing the prevalence of QTc prolongation and changes herein prospectively, with further view to assessing for the potential contribution of mutations in genes known to cause LQTS. The study aimed to relate findings to the phenotype, karyotype and the presence of cardiovascular malformations within the TS group. This study is the first to assess the QTcinterval in TS adhering to the recommendations of The American Heart Association using a linear function for QT adjustment of heart rate [13] and the first study to assess changes in the QTcinterval prospectively. 


\section{Methods}

\section{Ethics Statement}

The study was approved by the Ethics Committee of the Central Denmark Region (\#20010248) and registered at www. ClinicalTrials.gov (\# NGT00624949). All participants gave written informed consent.

\section{Study Subjects}

Women with karyotypically (Table 1$)$ proven TS $(n=102)$ with a mean follow-up of $4.7 \pm 0.5$ years were recruited through the Danish National Society of Turner Syndrome Contact Group and an endocrine outpatient clinic. The patients were examined at baseline, follow-up and end-of-study. Healthy, age-matched women $(n=68)$ were recruited by advertisement to serve as baseline controls. The controls were examined once.

\section{Electrocardiogram and QTc Measurements}

Twelve lead Electrocardiograms (EGG) were obtained using the Personal 120/210 machine (Esaote Biomedica, Cambridge, United Kingdom). All EGGs were recorded at $25 \mathrm{~mm} / \mathrm{s}$ with amplitude of $10 \mathrm{~mm} / \mathrm{mV}$. They were scanned to a digital file in 600 dpi and analyzed using Cardio Calipers 3.3 (Iconico, www. iconico.com) and measurements of RR and QT-interval were made on screen in lead II, V5 and V6. The U-wave was excluded using the "teach-the-tangent" method [13] and the bQTc-interval was calculated using Bazett's formula and hQTc-interval using Hodges's formula [14] in accordance with guidelines [13]. All ECGs were blinded and read by a single reader and the QTC established with an intra-observer variability of $0.7 \%$. A bQTcinterval with duration of less than 460 milliseconds (ms) was defined as normal [13].

With respect to women with TS, ECG recordings were initiated at first follow-up. At this point 3 women with TS had died, 8 were lost to follow-up and 26 did not have an ECG taken or the EGG was of poor quality. Before end of study in total 4 women with TS had died, 14 were lost to follow-up and 8 did not have an ECG or the ECG was of poor quality. In order to gain the greatest power we included all women with TS who had an ECG taken at either visit 2 or 3 in a pooled TS group using the most recent ECG in the final analysis $(\mathrm{n}=88)$. All controls had an ECG recording at baseline.

\section{Genetic Analysis}

In women with $\mathrm{TS}$ and a bQTc $>432 \mathrm{~ms}(\mathrm{~N}=40)$ we determined the presence of mutations in genes related to LQTS (KCNQ1, KCNH2, KCNE1, KCNE2, and SCN5A). DNA was extracted from EDTA stabilized blood using Maxwell ${ }^{\circledR} 16$ Blood
DNA Purification Kit. All coding exons including exon-intron boundaries of the genes KGNQ1, KGNH2, SCN5A, KGNE1, and KCNE2 were amplified and subsequently screened for mutations by High Resolution Melting Analysis using the LightScanner System (Idaho Technology Inc. Utah, USA), or by Sanger sequencing using a 3130XL Genetic Analyzer (Applied Biosystems). All primers for KCNQ1, KCNH2, KGNE1 and KCNE2 were designed in house. Primers for SCN5A were adapted from Millat [15], except for exons 2B, 5, $7,10,11,12 \mathrm{~A}, 13,16,17,18,20,22,27$, and 28E which were designed in-house. Primer sequences and PCR conditions are available upon request. All samples were analyzed for large genomic deletions by Multiplex Ligation dependent Probe Amplification (MLPA) using the SALSA MLPA kit P114 Long-QT (MRG Holland, Amsterdam, Holland). Mutations were classified according to the "Classifications System for Sequence Variants". [16].

\section{Laboratory Tests, Blood Pressures and Echocardiographic Assessment}

Blood samples (sodium, potassium, calcium, triglyceride, low density lipoprotein and haemoglobin A1G), 24-hour blood pressures and echocardiography were performed at baseline, follow-up and end-of-study in women with TS and at baseline in controls.

Echocardiographic assessment of aortic valve morphology was done by one experienced observer on a GE Vivid Seven (GE Healthcare, Horten, Norway) with a $2.5 \mathrm{MHz}$ transducer using second harmonic imaging. Images were obtained from parasternal and apical views during end-expiratory apnoea.

\section{Statistics}

Statistical computations were performed using SPSS 20.0. Continuous variables were expressed as means \pm standard deviations or medians (range) as appropriate. Variables were computed as absolute, log-transformed or inverse-transformed values, and compared by Student's independent t-test, Student's paired t-test, Mann-Whitney U-test or Wilcoxon signed rank test as appropriate. For binary variables Fisher's exact test was applied. Bivariate associations $(r)$ of continuous variables were tested using Pearsons coefficient of correlation, Spearman's rank correlation coefficient or partial correlation coefficients. Further explanatory models were constructed for the primary outcome parameter, QTcinterval length, using backwards multiple linear regression analyses. Independent variables were chosen from the baseline correlation analyses of continuous variables and dichotomous variables (i.e. bicuspid aortic valve and karyotype). Independent variables were omitted from the models when $\mathrm{P}>0.10$ and

Table 1. The karyotype distribution among woman with TS and an electrocardiogram.

\begin{tabular}{lcc}
\hline Karyotype & Percent \\
\hline $45, X$ & hQTc $>\mathbf{4 6 0}$ ms & 60.2 \\
Mosaic & 39.8 \\
$45, X / 46, X X / 47, X X X ; 45, X / 46, X X ; 45, X / 47, X X X$ & 3.4 \\
$45, X / 46, X, r(X) ;$ & 4.5 \\
Turner Mosaic with Y-material & 5.7 \\
Mosaic with isochromosom og dicentric chromosome & 19.3 \\
All other karyotypes & 1 of 17 \\
\hline doi:10.1371/journal.pone.0069614.t001 & 6.8
\end{tabular}


contributions to the final model were stated at standardized coefficients $(\beta)$. Otherwise, $\mathrm{P}<0.05$ was considered statistically significant.

The ECG findings are secondary outcomes from the original study assessing the aortic diameter by magnetic resonance imaging.

\section{Results}

The QT-interval was prolonged in women with TS irrespective of correction method (Table 2). Ten (Bazett's, $11 \% \mathrm{p}=0.005)$ and six (Hodges's, 7\% p = 0.036) of the 88 women with TS had a QTc $\geq 460 \mathrm{~ms}$. None of the controls had a prolonged QTc. Twenty percent of women with TS had a bQTc and 15\% a hQTc above $440 \mathrm{~ms}$. There were no differences over time in hQTc in the 51 women with TS seen at both first follow-up and last visit (416.9 \pm 22.6 vs. $415.6 \pm 25.5 ; p=0.4)$.

Heart rate was plotted against QTc to clarify the contribution of heart rate to differences in the QTc-interval (Figure 1A and B). There was a positive correlation between heart rate and bQTc among women with TS $(\mathrm{R}=0.448 ; \mathrm{p}<0.001)$ and controls $(\mathrm{R}=0.401 ; \mathrm{p}<0.001)$, which was not present using hQTc (Figure 1A and B). Since the use of Hodges formula effectively removed the dependence of QTc on heart rate, we chose to use
hQTc in all further calculations. A correlation was present between age and hQTc $(r=0.342 ; p=0.004)$ in controls, but not in women with TS (Table 3).

\section{Karyotype and Molecular Genetics}

The QTc was longer in women with TS and karyotype 45, X (Table 1) than all other karyotypes (hQTc 418.2 \pm 24.8 vs. 407.6 $\pm 25.5 ; \mathrm{p}=0.055)$. In women with $\mathrm{TS}$ and a bQTc $>432 \mathrm{~ms}(\mathrm{~N}=40)$ we analyzed for the presence of Long QT (LQT) mutations, and found 8 with mutations in one or more of the analyzed genes. We found 6 with a mutation in the KCNH2 gene (five with c.3140G $>\mathrm{T}$ heterozygous p.Arg1047Leu, rs36210421; one with c.2738C $>\mathrm{T}$ heterozygous p.Ala913Val), two with a mutation in SCN5A (one with c.5872C $>$ T p.Arg1958X; one with c.6010T $>$ C heterozygous p.Phe2004Leu) (Table 4). One woman with TS who was found to have a mutation in the KCNH2 gene also had a KCNE2 gene mutation (c.161T $>$ C heterozygous p.Met54Thr). The remaining 32 women with TS and the longest bQTc intervals did not have mutations in any of the studied genes. There was no difference neither in the bQT nor hQTc between those with and those without mutations (data not shown).

Table 2. Demographic, clinical and biochemical characteristics.

\begin{tabular}{|c|c|c|c|c|}
\hline & & \multirow{2}{*}{$\begin{array}{l}\text { Controls } \\
(n=68)\end{array}$} & \multirow{2}{*}{$\begin{array}{l}\text { Turner syndrome } \\
(n=88)\end{array}$} & \multirow[t]{2}{*}{ P-value } \\
\hline & & & & \\
\hline Bazett's QTc & [ms] & $389.1 \pm 20.1$ & $426.8 \pm 30.1$ & $<0.001$ \\
\hline Hodges's QTC & [ms] & $390.4 \pm 17.8$ & $414.0 \pm 25.5$ & $<0.001$ \\
\hline Heart rate & [bpm] & $59.45 \pm 9,05$ & $72.7 \pm 13.5$ & $<0.001$ \\
\hline Weight & {$[\mathrm{kg}]$} & $68.3 \pm 11.8$ & $59.1 \pm 14.1$ & $<0.001$ \\
\hline Height & {$[\mathrm{cm}]$} & $168.4 \pm 6.23$ & $147.0 \pm 7.10$ & $<0.001$ \\
\hline BMI & {$[\mathrm{kg} / \mathrm{m}]$} & $22.7(19.1-38.1)$ & $26.0(18.5-49.0)$ & $<0.001$ \\
\hline BSA & {$\left[\mathrm{m}^{2}\right]$} & $1.76 \pm 0.16$ & $1.51 \pm 0.18$ & $<0.001$ \\
\hline Age & [years] & $38.9 \pm 12.4$ & $42.3 \pm 10.4$ & 0.07 \\
\hline $24 \mathrm{~h}$ systolic BP & {$[\mathrm{mmHg}]$} & $112.7 \pm 10.5$ & $117.4 \pm 13.3$ & 0.02 \\
\hline $24 \mathrm{~h}$ diastolic BP & {$[\mathrm{mmHg}]$} & $71.2 \pm 8.0$ & $74.6 \pm 8.9$ & 0.02 \\
\hline $24 \mathrm{~h}$ heart rate & [bpm] & $71.4 \pm 9.0$ & $74.5 \pm 10.6$ & 0.053 \\
\hline $24 \mathrm{~h}$ heart rate daytime & [bpm] & $74.7 \pm 9.2$ & $77.6 \pm 11.0$ & 0.09 \\
\hline $24 \mathrm{~h}$ heart rate nighttime & {$[\mathrm{bpm}]$} & $62.3 \pm 8.6$ & $67.8 \pm 10.5$ & 0.001 \\
\hline Dipping & [\%] & $14.8 \pm 4.37$ & $13.4 \pm 6.92$ & 0.1 \\
\hline Hypertensive treatment & [\%] & $0 \pm 0$ & $53.4 \pm 0.50$ & $<0.001$ \\
\hline HRT & [\%] & $0 \pm 0$ & $81.8 \pm 0.39$ & $<0.001$ \\
\hline Statins prescribed & [\%] & $0 \pm 0$ & $12.5 \pm 0.33$ & $<0.003$ \\
\hline Diabetes & [\%] & $0 \pm 0$ & $12.5 \pm 0.33$ & $<0.003$ \\
\hline Calcium ion & {$[\mathrm{mmol} / \mathrm{l}]$} & $1.22 \pm 0.04$ & $1.21 \pm 0.04$ & 0.057 \\
\hline Sodium & {$[\mathrm{mmol} / \mathrm{l}]$} & $139.6 \pm 1.87$ & $139.8 \pm 2.04$ & 0.5 \\
\hline Potassium & {$[\mathrm{mmol} / \mathrm{l}]$} & $3.73 \pm 0.23$ & $3.77 \pm 0.26$ & 0.3 \\
\hline LDL & {$[\mathrm{mmol} / \mathrm{l}]$} & $2.88 \pm 0.85$ & $2.90 \pm 0.77$ & 0.9 \\
\hline Triglyceride & {$[\mathrm{mmol} / \mathrm{l}]$} & $0.90(0.4-4.5)$ & $0.95(0.4-2.6)$ & 0.3 \\
\hline $\mathrm{HbA1C}$ & [\%] & $5.30(4.6-6.6)$ & $5.3(4.5-8.6)$ & 0.3 \\
\hline
\end{tabular}

Values are expressed as mean \pm standard deviation or median (minimum-maximum).

$\mathrm{BMI}=$ body mass index, $\mathrm{BSA}=$ body surface area, $\mathrm{BP}=$ blood pressure, $\mathrm{LDL}=$ low density lipoprotein, $\mathrm{HbA} 1 \mathrm{C}=\mathrm{Hemoglobin} \mathrm{A} 1 \mathrm{C}, \mathrm{ms}=\mathrm{milliseconds}, \mathrm{bpm}=$ beats per minute, NA = Not assessed, $\mathrm{HRT}=$ Hormone replacement therapy.

P-values $<0.05$ are considered statistically significant.

doi:10.1371/journal.pone.0069614.t002 


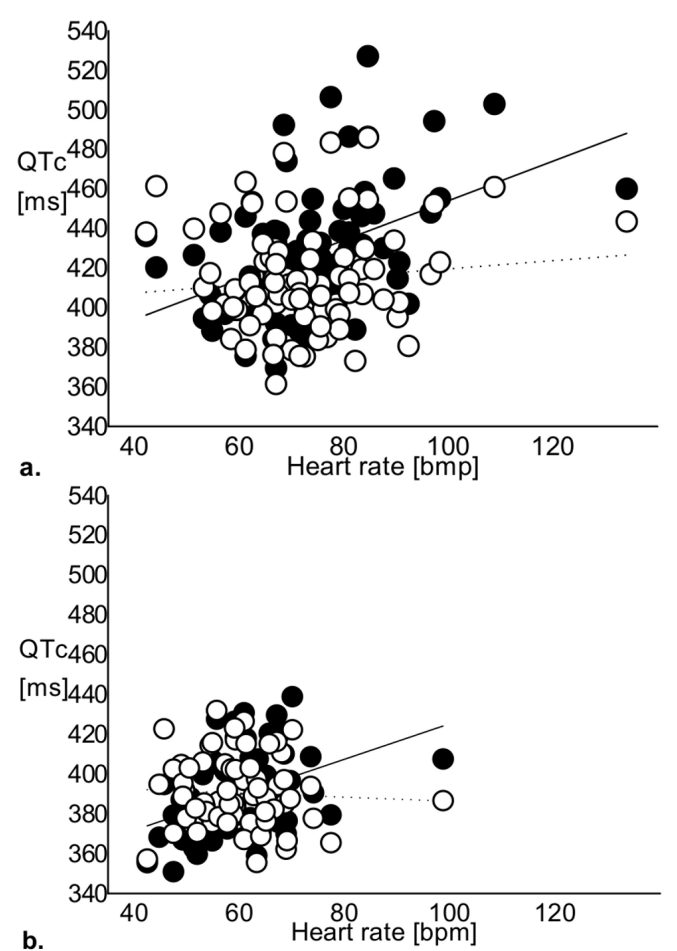

Figure 1. Heart rate plotted against QTc length. a. Heart rate plotted against QTc length in women with TS. Hodges's formula (open circles, dashed regression line, $r^{2}=0.012 ; p=0.3$ ) effectively omitted the dependence of QTc on heart rate when compared to Bazett's formula (filled circles, solid regression line, $r^{2}=0.200 ; p<0.001$ ). b. Heart rate plotted against QTc length in controls. Hodges's formula (open circles, dashed regression line, $r^{2}=0.003 ; p=0.7$ ) effectively omitted the dependence of QTc on heart rate when compared to Bazett's formula (filled circles, solid regression line, $r^{2}=0.161 ; p=0.001$ ). $\mathrm{ms}=$ milliseconds, bpm $=$ beats per minute.

doi:10.1371/journal.pone.0069614.g001

\section{Bicuspid Aortic Valve and QTC}

The presence or absence of bicuspid aortic valve was not associated with hQTc prolongation in women with TS (418.6 \pm 23.9 vs. $411.7 \pm 26.0 ; p=0.3$ ).

\section{QTc Correlations and Multiple Regression Analysis}

We then went on to study the dependent variable hQTc in backward multiple linear regression analyses, including variables that had proven to be predictors of hQTc in bivariate correlation analyses (Table 3) and which theoretically could have an effect on the QTc-interval. In one such model daytime heart rate $(\beta=-0.001 ; \mathrm{p}<0.001)$, calcium ion $(\beta=-0.125 ; \mathrm{p}=0.059)$, potassium $(\beta=-0.023 ; p=0.01)$, sodium $(\beta=-0.004 ; p=0.01)$, BMI $(\beta=0.001 ; p=0.02)$, and age $(\beta=0.0005 ; p<0.001)$ were independent explanatory variables of hQTc in women with TS. Variables obtained from medicinal care records, 24-hour blood pressures and other serum markers did not prove to be of use. In controls explanatory variables of hQTc were age $(\beta=0.001$; $\mathrm{p}<0.001)$, daytime heart rate $(\beta=-0.001 ; \mathrm{p}<0.001)$, and calcium ion $(\beta=-0.093 ; \mathrm{p}=0.052)$.

As mentioned four women with TS died prior to having an ECG taken for the purpose of the study. One died following operation of an aortic aneurism (hQTc $=428.8 \mathrm{~ms}$ ), one due to non-witnessed epileptic seizure $(\mathrm{hQTc}=435.2 \mathrm{~ms})$, one from unexpected cardiac arrest 5 days after a colon resection
Table 3. Bivariate correlations with hQTc.

\begin{tabular}{|c|c|c|c|c|c|}
\hline & & \multicolumn{2}{|c|}{ Controls } & \multicolumn{2}{|c|}{ Turner syndrome } \\
\hline & & hQTc & & hQTc & \\
\hline Age & [years] & 0.342 & $(0.004)$ & 0,204 & $(0.06)$ \\
\hline BMI & {$[\mathrm{kg} / \mathrm{m}]$} & 0,206 & $(0.09)$ & 0.089 & $(0.4)$ \\
\hline Heart rate day time & [bpm] & -0.390 & $(0.001)$ & -0.142 & $(0.2)$ \\
\hline Heart rate night time & [bpm] & -0.280 & $(0.02)$ & -0.053 & $(0.6)$ \\
\hline Calcium lon & {$[\mathrm{mmol} / \mathrm{l}]$} & -0.141 & $(0.3)$ & -0.232 & $(0.03)$ \\
\hline Potassium & {$[\mathrm{mmol} / \mathrm{l}]$} & 0.098 & $(0.4)$ & -0.192 & $(0.07)$ \\
\hline Sodium & {$[\mathrm{mmol} / \mathrm{l}]$} & 0.164 & $(0.2)$ & -0.269 & $(0.01)$ \\
\hline Karyotype: Mosaic Vs.45,X & & & & 0.299 & $(0.03)$ \\
\hline
\end{tabular}

Values are expressed as Spearman's Correlations coefficients (P-value)

$\mathrm{BMI}=$ body mass index, $\mathrm{bpm}=$ beats per minute.

$P$-values $<0.05$ are considered statistically significant.

doi:10.1371/journal.pone.0069614.t003

(hQTc $=402.7 \mathrm{~ms}$ ) and the last was found dead in her home (no ECG the patient file).

\section{Discussion}

The principal results from our study provide evidence that mutations in LQTS genes are highly prevalent in adult women with TS and a QTc $>432 \mathrm{~ms}$. We speculate that these findings may be one of numerous possible mechanisms behind QTc prolongation in TS. However, this study does not answer the question as to what extent mutations in LQTS genes may explain a proportion of the unexplained excess mortality which is well known in Turner cohorts $[9,10]$.

Moreover, we assessed the QTc-interval prospectively since a prolongation of the QTc-interval over time could suggest acquired LQT due to e.g. medication prescribed to treat comorbidities. However the QTc-interval did not change during the follow-up period.

Previous studies of QTc in TS have used Bazett's formula which retains a strong residual correlation with heart rate [13], as shown here. According to guidelines [13] we estimated QTc using a linear approach (Hodges's formula) as well as Bazett's formula, and results depend on the method used. Our use of both equations makes comparisons with previous studies feasible and Hodges's formula effectively eliminates any residual correlation with heart rate. We found the prevalence of QTc above $440 \mathrm{~ms}$ (bQTc $20 \%$ and hQTc 15\%) comparable with previous studies (21-36\%) [13]. We suggest future use of Hodges's formula when estimating QTc as to remove the dependence of heart rate.

In the general population $4 \%$ of Caucasians may have a LQTS positive gene test [6], which markedly contrasts with the prevalence of $20 \%$ of women with TS in the genetically screened group resulting in a prevalence of at least $9 \%$ in the complete TS cohort. In the TS cohort $7.5 \%$ of the mutations where known pathogenic mutations. We have no genetic explanation for the apparent co-segregation of LQT mutations with the complete or partial loss of an X in TS.

Seven of the 8 LQT mutations in the present study were missense mutations and one resulted in a stop codon. KCNH2 c. $3140 \mathrm{G}>\mathrm{T}$ is often reported as a polymorphism without detectable differences in channel function when compared to the wild type $[17,18]$ though not consistently [19-21]. A prevalence of $4 \%$ has been reported in a Danish cohort [21]. It has been speculated that KCNH2 c.3140G $>$ T may be associated with 
Table 4. Long QT mutation carriers' descriptives.

\begin{tabular}{|c|c|c|c|c|c|c|c|c|}
\hline \multirow[t]{2}{*}{ Mutation } & \multirow[t]{2}{*}{$\mathrm{KCNH}^{4}$} & $\mathrm{KCNH}^{1}{ }^{1}$ & $\mathrm{KCNH}^{1}$ & $\mathrm{KCNH}^{1}$ & $\mathrm{KCNH}^{1}{ }^{1}$ & SCN5A ${ }^{2}$ & SCN5A $^{3}$ & $\mathrm{KCNH}^{2}{ }^{1}$ \\
\hline & & \multicolumn{7}{|l|}{$\mathrm{KCNE2}^{5}$} \\
\hline De novo/inherited & Mater & $?$ & Pater & $?$ & $?$ & Pater & Mater & $?$ \\
\hline \multirow[t]{2}{*}{ Karyotype } & $45, X$ & $45, X$ & $45, X$ & $45, X / 46, X, i(X q)$ & $45, X, \operatorname{inv}(9)$ & $45, X$ & $45, X /$ & $45, x$ \\
\hline & & & & $47, X, i(X q), i(X q)$ & (p11q11) & & $46, X, \operatorname{idic}(X)$ & \\
\hline Bazett's QTc [ms]* & 468.7 & 465.2 & 464.8 & 458.3 & 453.5 & 453.4 & 443.4 & 432.0 \\
\hline Hodges's QTc [ms]* & 444.3 & 433.9 & 435.8 & 434.6 & 427.6 & 452.6 & 424.8 & 415.6 \\
\hline Heart rate [bmp] & 68 & 90 & 134 & 71 & 79 & 62 & 65 & 79 \\
\hline Bicuspid aortic valve & Yes & No & No & No & No & Yes & No & Yes \\
\hline Coarctatio & No & No & Yes & No & No & Yes & No & No \\
\hline Hypothyroidisme & No & Eltroxin & Eltroxin & No & No & Eltroxin & No & No \\
\hline Ischemic heart disease & No & No & No & No & No & No & No & No \\
\hline Diabetes mellitus & No & No & No & Yes & No & No & No & No \\
\hline Hypertension & No & Yes & Yes & Yes & No & Yes & No & Yes \\
\hline Antihypertensive drugs & No & Yes & Yes & Yes & Yes & Yes & Yes & No \\
\hline Age [years] & 26 & 56 & 43 & 42 & 32 & 41 & 38 & 38 \\
\hline HRT during entire study & No & Yes & Yes & Yes & Yes & Yes & Yes & Yes \\
\hline $\mathrm{BMI}>30$ & No & Yes & Yes & Yes & No & Yes & Yes & No \\
\hline Treatment for LQTS & No & No & $?$ & $\beta$-blocker & $\beta$-blocker & No & $?$ & $\beta$-blocker \\
\hline
\end{tabular}

*ECGs with the longest QTc-interval.

${ }^{1} \mathrm{KCNH} 2$ c.3140G > T heterozygous p.Arg1047Leu. Uncertain pathogenic, known mutation.

${ }^{2} \mathrm{SCN} 5 \mathrm{~A}$ c.6010T $>$ C heterozygous p.Phe2004Leu. Uncertain pathogenic, known mutation.

${ }^{3}$ SCN5A c.5872C $>$ T heterozygous p.Arg1958X. Definitely pathogenic, Stop codon mutation.

${ }^{4} \mathrm{KCNH} 2$ c.2738C $>$ T heterozygous p.Ala913Val. Definitely pathogenic.

${ }^{5}$ KCNE2 C.161T $>C$ heterozygous p.Met54Thr. Likely pathogenic.

A question mark indicates that no information was available. $\mathrm{ms}=$ milliseconds, $\mathrm{bpm}=$ beats per minute. HRT $=$ Hormone replacement therapy.

The in silico analysis was perfomed by the use of Alamut software (Interactive Biosoftware, ver 2.1, Mont-Saint-Aignan, France, http://www.interactive-biosoftware.com/)

taking into account results from Polyphen-2, SIFT, Grantham score, and the nucleotide and aminoacid conservation.

doi:10.1371/journal.pone.0069614.t004

particular susceptibility towards hypokalemia or certain drugs, as it seems the case in hypokalemic patients with C-terminal mutations in HERG [22]. Lastly, it has been reported in patients with Torsade des Pointes ventricular tachycardia [23]. The SCN5A c.5872C $>\mathrm{T}$ results in a stop codon mutation. The SCN5A c.6010T $>\mathrm{C}$ mutation has been linked to sudden infant death [24] and increased persistent sodium current [24,25]. In addition, depolarizing shifts in voltage dependence of inactivation and faster recovery from inactivation [25] have been described. KCNE2 c. $161 \mathrm{~T}>\mathrm{C}$ has been related to exercise induced ventricular tachycardia [26], Torsade des Pointes ventricular tachycardia [27], diminished potassium flux, a less readily activation and a more rapid deactivation. The latter gene mutation has also been encountered in procainamide-induced LQTS and carriers of this mutation have longer QTc-intervals. [28] KCHN2 c.2738C > T has been reported to lead to the Romano-Ward syndrome [29,30]. Considering the in silico prediction results the mutations were classified as shown in Table 4.

Our study confirmed a negative correlation with ionized calcium and potassium but also sodium in TS but opposed to the healthy controls. Interestingly, mean values of calcium, sodium and potassium were comparable with controls and within normal limits. Calcium was the only variable with an impact on the hQTc-interval in our multiple regression analysis. Prior studies have explored differences in electrolytes between women with TS and controls as well as groups with TS and prolonged versus normal QTc. However, an actual correlation between the electrolytes and the QTc is a novel finding [1-3,31]. A correlation between QTc and calcium within the reference interval has been described in non-TS patients with cirrhosis [32] and with serum sodium [33]. Whether repolarization in women with TS (and thus the resultant QTc-interval) is more sensitive to changes in electrolytes within the normal range is not known.

The aetiology of the altered cardiac electrophysiology in TS is unknown but several aspects suggest an inherited defect. In accordance with Dalla Pozza et al [3], but in contrast to others $[1,2]$, we found the $45, \mathrm{X}$ karyotype to have an adverse impact on the heart rate corrected QT-interval. Secondly the hQTc-interval prolongation is not associated with traditional indices of QTcinterval elongation (i.e. age, left ventricular hypertrophy, medicinal use, coronary heart disease and thyroid disorders) $[1,2]$. Bicuspid aortic valve had no influence on hQTc, a finding confirmed by previous studies. [1,2].

Gender affects QTc as is evident through testosterone mediated shortening of QTc-intervals in males [34,35]. Endogenous as well as exogenous progesterone also shorten QTc [36] and counteracts a QTc prolonging effect of exogenous estrogen [35,36] and therefore HRT does not affect QTc [37]. Low endogenous progesterone and androgens as well as unopposed estrogen therapy could therefore in women with TS, at least on a theoretical level, prolong QTc-intervals.

The length of the QTc-interval in women with TS differs from that of age matched girls with short stature. [3] It remains to be resolved, if the X-chromosomal haploinsufficiency in women with TS results in altered transcription of proteins involved in the activity of ion channels as structural or regulatory components, 
such as is seen in congenital LQTS [38]. Alternatively, altered expression of $\mathrm{X}$-linked genes may function as transcriptional regulators of autosomal genes involved in ion channel activity. Interestingly, the action potential of cardiomyocyte-like differentiated pluripotent stem cells is prolonged in women with TS [39], which points towards a primary developmental defect. Our multiple regression analysis explained less than $30 \%$ of the variation in the hQTc-interval length. Further insight is necessary into the aetiology of QTc prolongation, including the role played by the relatively increased sympathetic drive in women with TS $[40,41]$ that likely increases automaticity, excitability and conductivity of cardiomyocytes and cardiac conduction tissues.

Drug therapy, including HRT, antihypertensive and cholesterol lowering drugs was not correlated with hQTc in this cohort. Interestingly, the empiric recommendation of beta-blockers to treat aortic dilatation [42] may have been beneficial in numerous cases, since beta-blockers are the drug of choice in patients with TS [43].

\section{Limitations}

Since differing height and body composition is inherent in women with TS we included BMI and BSA as a confounder in our calculations instead of choosing a control group matching the former mentioned criteria since such controls cannot be considered entirely healthy.

No information on the plasma levels of sex hormones was available. Yet it can be noted that no difference was demonstrated between patients with or without HRT.

We screened women with TS and a bQTc $>432.0 \mathrm{~ms}$ and no controls. Hence there is the possibility that the controls harbour the same prevalence of mutations or that the women with TS who were not screened for mutations had a different prevalence of LQT mutations.

\section{References}

1. Bondy CA, Ceniceros I, Van PL, Bakalov VK, Rosing DR (2006) Prolonged rate-corrected QT interval and other electrocardiogram abnormalities in girls with Turner syndrome. Pediatrics 118: e1220-e1225.

2. Bondy CA, Van PL, Bakalov VK, Sachdev V, Malone CA, et al. (2006) Prolongation of the cardiac QTc interval in Turner syndrome. Medicine (Baltimore) 85: 75-81.

3. Dalla Pozza R, Bechtold S, Kaab S, Buckl M, Urschel S, et al. (2006) QTc interval prolongation in children with Ulrich-Turner syndrome. Eur J Pediatr 165: 831-837.

4. Kannel WB, Kannel C, Paffenbarger RS Jr, Cupples LA (1987) Heart rate and cardiovascular mortality: the Framingham Study. AmHeart J 113: 1489-1494.

5. Haverkamp W, Breithardt G, Camm AJ, Janse MJ, Rosen MR, et al. (2000) The potential for QT prolongation and proarrhythmia by non-antiarrhythmic drugs: clinical and regulatory implications. Report on a policy conference of the European Society of Cardiology. Eur Heart J 21: 1216-1231.

6. Kapa S, Tester DJ, Salisbury BA, Harris-Kerr C, Pungliya MS, et al. (2009) Genetic testing for long-QT syndrome: distinguishing pathogenic mutations from benign variants. Circulation 120: 1752-1760.

7. Gravholt $\mathrm{CH}$ (2004) Epidemiological, endocrine and metabolic features in Turner syndrome. EurJEndocrinol 151: 657-687.

8. Gravholt CH, Juul S, Naeraa RW, Hansen J (1998) Morbidity in Turner syndrome. J ClinEpidemiol 51: 147-158.

9. Schoemaker MJ, Swerdlow AJ, Higgins CD, Wright AF, Jacobs PA (2008) Mortality in women with turner syndrome in Great Britain: a national cohort study. J ClinEndocrinolMetab 93: 4735-4742.

10. Stochholm K, Juul S, Juel K, Naeraa RW, Gravholt CH (2006) Prevalence, incidence, diagnostic delay, and mortality in Turner syndrome. J ClinEndocrinolMetab 91: 3897-3902.

11. Sozen AB, Cefle K, Kudat H, Ozturk S, Oflaz H, et al. (2008) Atrial and ventricular arryhthmogenic potential in Turner Syndrome. Pacing ClinElectrophysiol 31: 1140-1145.

12. Golzio PG, Franco E, Chiribiri A (2006) Atrio-ventricular synchronization by single VDD lead inserted through persistent left superior vena cava in patient with Turner's syndrome. Pacing Clin Electrophysiol 29: 1181-1182.

13. Rautaharju PM, Surawicz B, Gettes LS, Bailey JJ, Childers R, et al. (2009) AHA/ACCF/HRS recommendations for the standardization and interpretation of the electrocardiogram: part IV: the ST segment, T and U waves, and the QT

\section{Future Perspectives}

We anticipate that future studies will provide more knowledge about the genetic and epigenetic profile of women with TS, aspects of inheritance as well as the effect of androgens and progesterone. Use of Hodges's formula to estimate the QTc should be recommended to eliminate the influence of heart rate. In the clinical care of women with TS, the clinician should be aware of LQTS. Annual ECGs and on a regularly basis evaluation by a cardiologist is paramount. Beta-blockers may be prescribed as first choice antihypertensive drug targeting hypertension as well as LQTS. Care should be taken to avoid drugs known to lengthen the QTc-interval and if long-term unopposed estrogen therapy is prescribed, it should be followed by regular reevaluation of the QTc-interval.

\section{Conclusion}

There is a high prevalence of mutations in the major LQTS genes in women with TS and prolonged QTc. However, it remains to be settled, whether these findings are related to the unexplained excess mortality in Turner women.

\section{Acknowledgments}

Lone Kvist and Merete Møller are thanked for their expert technical help.

\section{Author Contributions}

Conceived and designed the experiments: CHG KHM NHA. Performed the experiments: KHM NHA LNP. Analyzed the data: CT CHG KHM NHA HKJ AB LNP. Contributed reagents/materials/analysis tools: CT LNP. Wrote the paper: CT CHG KHM NHA HKJ AB LNP interval: a scientific statement from the American Heart Association Electrocardiography and Arrhythmias Committee, Council on Clinical Cardiology; the American College of Cardiology Foundation; and the Heart Rhythm Society: endorsed by the International Society for Computerized Electrocardiology. Circulation 119: e241-250.

14. Hodges M (1983) Bazett's OT correction reviewed-Evidence that a linear OT correction for heart is better. J Am Coll Cardiol 12: 694.

15. Millat G, Chanavat V, Rodriguez-Lafrasse C, Rousson R (2009) Rapid, sensitive and inexpensive detection of SCN5A genetic variations by high resolution melting analysis. ClinBiochem 42: 491-499.

16. Plon SE, Eccles DM, Easton D, Foulkes WD, Genuardi M, et al. (2008) Sequence variant classification and reporting: recommendations for improving the interpretation of cancer susceptibility genetic test results. Hum Mutat 29: 1282-1291.

17. Anson BD, Ackerman MJ, Tester DJ, Will ML, Delisle BP, et al. (2004) Molecular and functional characterization of common polymorphisms in HERG (KCNH2) potassium channels. Am J Physiol Heart Circ Physiol 286: H2434 2441

18. Marjamaa A, Newton-Cheh C, Porthan K, Reunanen A, Lahermo P, et al. (2009) Common candidate gene variants are associated with QT interval duration in the general population. J Intern Med 265: 448-458.

19. Sun Z, Milos PM, Thompson JF, Lloyd DB, Mank-Seymour A, et al. (2004) Role of a KCNH2 polymorphism (R1047 L) in dofetilide-induced Torsades de Pointes. J Mol Cell Cardiol 37: 1031-1039.

20. Chevalier P, Bellocq C, Millat G, Piqueras E, Potet F, et al. (2007) Torsades de pointes complicating atrioventricular block: evidence for a genetic predisposition. Heart Rhythm 4: 170-174.

21. Larsen LA, Andersen PS, Kanters J, Svendsen IH, Jacobsen JR, et al. (2001) Screening for mutations and polymorphisms in the genes KCNH2 and KCNE2 encoding the cardiac HERG/MiRP1 ion channel: implications for acquired and congenital long Q-T syndrome. Clin Chem 47: 1390-1395.

22. Berthet M, Denjoy I, Donger C, Demay L, Hammoude H, et al. (1999) Gterminal HERG mutations: the role of hypokalemia and a KCNQ1-associated mutation in cardiac event occurrence. Circulation 99: 1464-1470.

23. Mank-Seymour AR, Richmond JL, Wood LS, Reynolds JM, Fan YT, et al. (2006) Association of torsades de pointes with novel and known single nucleotide polymorphisms in long QT syndrome genes. Am Heart J 152: 1116-1122. 
24. Arnestad M, Crotti L, Rognum TO, Insolia R, Pedrazzini M, et al. (2007) Prevalence of long-QT syndrome gene variants in sudden infant death syndrome. Circulation 115: 361-367.

25. Wang DW, Desai RR, Crotti L, Arnestad M, Insolia R, et al. (2007) Cardiac sodium channel dysfunction in sudden infant death syndrome. Circulation 115: 368-376.

26. Abbott GW, Sesti F, Splawski I, Buck ME, Lehmann MH, et al. (1999) MiRP1 forms $\mathrm{IKr}$ potassium channels with HERG and is associated with cardiac arrhythmia. Cell 97: 175-187.

27. Paulussen AD, Gilissen RA, Armstrong M, Doevendans PA, Verhasselt P, et al. (2004) Genetic variations of KCNQ1, KCNH2, SCN5A, KCNE1, and KCNE2 in drug-induced long OT syndrome patients. J Mol Med (Berl) 82: 182-188.

28. Sesti F, Abbott GW, Wei J, Murray KT, Saksena S, et al. (2000) A common polymorphism associated with antibiotic-induced cardiac arrhythmia. Proc Natl Acad Sci U S A 97: 10613-10618.

29. Kapplinger JD, Tester DJ, Salisbury BA, Carr JL, Harris-Kerr C, et al. (2009) Spectrum and prevalence of mutations from the first 2,500 consecutive unrelated patients referred for the FAMILION long QT syndrome genetic test. Heart Rhythm 6: 1297-1303

30. Tester DJ, Will ML, Haglund CM, Ackerman MJ (2005) Compendium of cardiac channel mutations in 541 consecutive unrelated patients referred for long QT syndrome genetic testing. Heart Rhythm 2: 507-517.

31. Dalla Pozza R, Bechtold S, Urschel S, Netz H, Schwarz HP (2009) QTc interval prolongation in children with Turner syndrome: the results of exercise testing and 24-h ECG. EurJ Pediatr 168: 59-64.

32. Genovesi S, Prata Pizzala DM, Pozzi M, Ratti L, Milanese M, et al. (2009) QT interval prolongation and decreased heart rate variability in cirrhotic patients: relevance of hepatic venous pressure gradient and serum calcium. Clin Sci (Lond) 116: 851-859.

33. Sohaib SM, Papacosta O, Morris RW, Macfarlane PW, Whincup PH (2008) Length of the QT interval: determinants and prognostic implications in a population-based prospective study of older men. J Electrocardiol 41: 704-710.
34. Pecori Giraldi F, Manzoni G, Michailidis J, Scacchi M, Stramba-Badiale M, et al. (2011) High prevalence of prolonged QT interval in obese hypogonadal males. Obesity (Silver Spring) 19: 2015-2018.

35. Rautaharju PM, Zhou SH, Wong S, Calhoun HP, Berenson GS, et al. (1992) Sex differences in the evolution of the electrocardiographic QT interval with age. Can J Cardiol 8: 690-695.

36. Rodriguez I, Kilborn MJ, Liu XK, Pezzullo JC, Woosley RL (2001) Druginduced QT prolongation in women during the menstrual cycle. JAMA 285: $1322-1326$.

37. Kadish AH, Greenland P, Limacher MC, Frishman WH, Daugherty SA, et al. (2004) Estrogen and progestin use and the QT interval in postmenopausal women. Ann Noninvasive Electrocardiol 9: 366-374.

38. Bokil NJ, Baisden JM, Radford DJ, Summers KM (2010) Molecular genetics of long QT syndrome. MolGenetMetab 101: 1-8.

39. Li W, Wang X, Fan W, Zhao P, Chan YC, et al. (2012) Modeling abnormal early development with induced pluripotent stem cells from aneuploid syndromes. HumMolGenet 21: 32-45.

40. Gravholt CH, Hansen KW, Erlandsen M, Ebbehoj E, Christiansen JS (2006) Nocturnal hypertension and impaired sympathovagal tone in Turner syndrome. J Hypertens 24: 353-360.

41. Zuckerman-Levin N, Zinder O, Greenberg A, Levin M, Jacob G, et al. (2006) Physiological and catecholamine response to sympathetic stimulation in turner syndrome. Clin Endocrinol (Oxf) 64: 410-415.

42. Erbel R, Alfonso F, Boileau G, Dirsch O, Eber B, et al. (2001) Diagnosis and management of aortic dissection. Eur Heart J 22: 1642-1681.

43. Zipes DP, Camm AJ, Borggrefe M, Buxton AE, Chaitman B, et al. (2006) ACC/ AHA/ESC 2006 guidelines for management of patients with ventricular arrhythmias and the prevention of sudden cardiac death: a report of the American College of Cardiology/American Heart Association Task Force and the European Society of Cardiology Committee for Practice Guidelines (Writing Committee to Develop Guidelines for Management of Patients With Ventricular Arrhythmias and the Prevention of Sudden Cardiac Death). J Am Coll Cardiol 48: e247-346. 